\title{
NGHIÊN CỬU XÁC ĐỊNH SỐ BẬC TỐI ĐA CỦA MÔ HÌNH HẸ SỐ ĐIỀU HÒA CÂU TRONG THỰC TẾ TÍNH TOÁN DI THƯỜNG ĐỘ CAO TRÊN LÃNH THỔ VIẸT NAM
}

\author{
ThS. NGUYẼ̃N TUÂN ANH \\ Viện Khoa học Đo đạc và Bản đồ
}

\section{Tóm tắt:}

Bài báo khoa học phân tích quá trình xây dựng mô hình các hệ số điều hòa cầu từ các nguồn dữ liệu khác nhau và thực nghiệm tính toán giá trị dị thường độ cao tại khu vực Việt Nam theo sự thay đổi bậc tối đa của các hệ số điều hòa cầu. Kết quả nhận được cho thấy do chưa sử dụng dữ liệu đo trọng lực chi tiết, mô hình hệ số điều hòa cầu chỉ hiệu quả đến bậc khai triển 180.

\section{1. Đặt vấn đề}

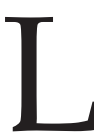
ực hấp dẫn tạo nên thế năng tại 1 điểm trên bề mặt Trái Đất là một đại lượng vật lý tự nhiên tồn tại khách quan dưới sự tác động hỗn hợp bởi lực li tâm do chuyển động quay của Trái Đất, lực hấp dẫn do các đối tượng vật chất phân bố trong lòng Trái Đất và lực hấp dẫn bởi các hành tinh trong vũ trụ. Tuy nhiên bằng việc đo đạc trọng lực tại các điểm khác nhau phân bố trên Trái Đất các nhà khoa học đã cố gắng xây dựng được mô hình các hệ số điều hòa cầu làm tham số cho việc tính toán, mô tả được thế trọng trường Trái Đất bằng hàm toán học (Hàm điều hòa cầu). Những nỗ lực ban đầu được đề xuất bởi Zhongolovich 1956 [2] có thể đưa ra mô hình hệ số điều hòa cầu đến bậc thứ 8 từ một số điểm đo trọng lực mặt đất. Sau đó với sự phát triển của công nghệ vệ tinh, ban đầu người ta thấy rằng các ảnh hưởng của trọng trường Trái Đất làm chệch hướng quỹ đạo vệ tinh bay quanh Trái Đất, như vậy bằng các phép phân tích và tính toán tương quan cho phép xác định được trường trọng lực Trái Đất từ vệ tinh với độ bao phủ tốt hơn từ đó có thể xác định được các hệ số điều hòa cầu từ dữ liệu vệ tinh [2]. Các dữ liệu trọng lực vệ tinh cho phép xác định trường trọng lực Trái Đất với bước sóng dài ở đó bậc tối đa của các hệ số điều hòa cầu từ 4 vào năm 1960 đến 180 cho tới ngày nay khi các dữ liệu đo vệ tinh ngày càng hoàn thiện.

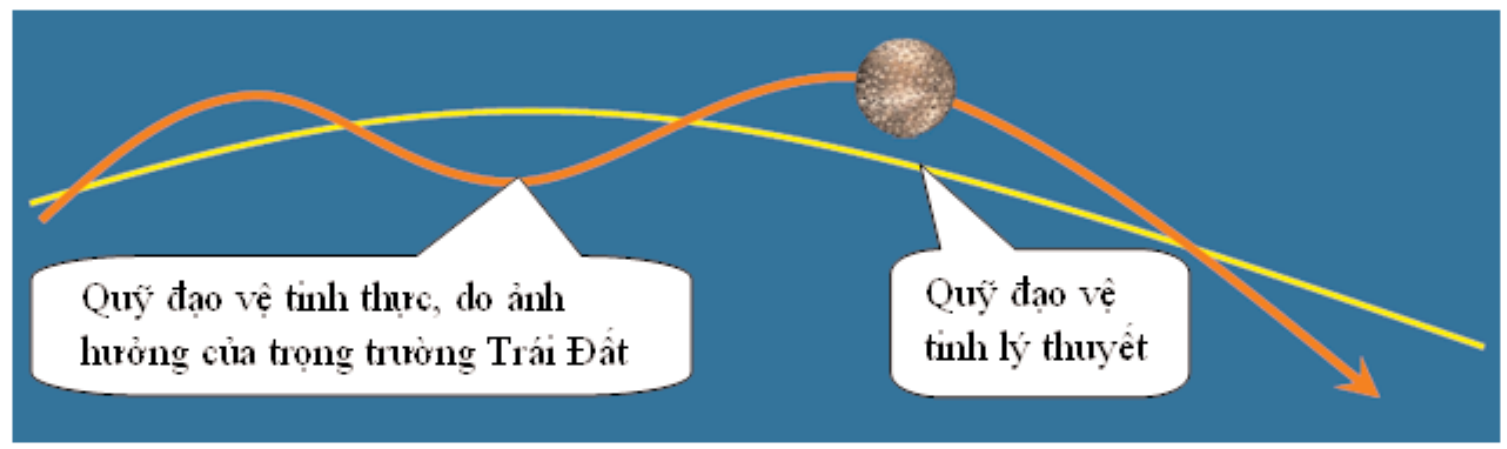

Hình 1: Ảnh hưởng nhiễu quỹ đạo vệ tinh do trọng trường Trái Đất

Người phản biện: TS. Dương Chí Công 
Để xác định được các thông tin bước sóng ngắn có mật độ cao người ta đã sử dụng giải pháp kết hợp giữa dữ liệu trọng lực vệ tinh và trọng lực mặt đất, với rất nhiều các thuật toán được đưa ra để áp dụng cho việc kết hợp này nhằm mô phỏng được chi tiết và chính xác nhất thế trọng trường Trái Đất bằng các hệ số hàm điều hòa cầu. Một vài mô hình kết hợp với bậc tối đa là 180 hoặc cao hơn đã được phát triển từ năm 1978 [2], các mô hình khác từ năm 1981 như OSU1981 [2] và GEM 10c [2]. Từ năm 1985 đã hoàn thiện tới bậc 360 có GMP2 [2], Rapp and Cruz [2], OSU86 E/F.

Một vài năm gần đây đã có một số điều kiện thuận lợi cho việc mở rộng các hệ số điều hòa cầu với số bậc, độ chính xác và mức độ chi tiết cao hơn. Việc phát triển đó dựa trên cơ sở cải thiện mô hình lý thuyết, tăng độ chính xác, hoàn thiện dữ liệu trọng lực vệ tinh (GRACE, GOCE), tăng dày và mở rộng dữ liệu trọng lực mặt đất, tăng cường dữ liệu đo cao vệ tinh, đặc biệt là việc chuẩn hóa và xử lý đồng bộ trên tập dữ liệu lớn từ nhiều nguồn khác nhau cho phép xác định các hệ số điều hòa cầu đến bậc 2190 (EGM2008) [1].

Đến nay mô hình hệ số điều hòa cầu hoàn chỉnh nhất được cung cấp chính thức cho người sử dụng trên toàn cầu là mô hình EGM2008 có bậc tối đa lên tới 2190, trong mô hình này các nguồn dữ liệu đưa vào để tính toán các hệ số điều hòa cầu rất đa dạng gồm trọng lực mặt đất, trọng lực hàng không, đo cao vệ tinh, trọng lực từ dự án cặp vệ tinh GRACE.

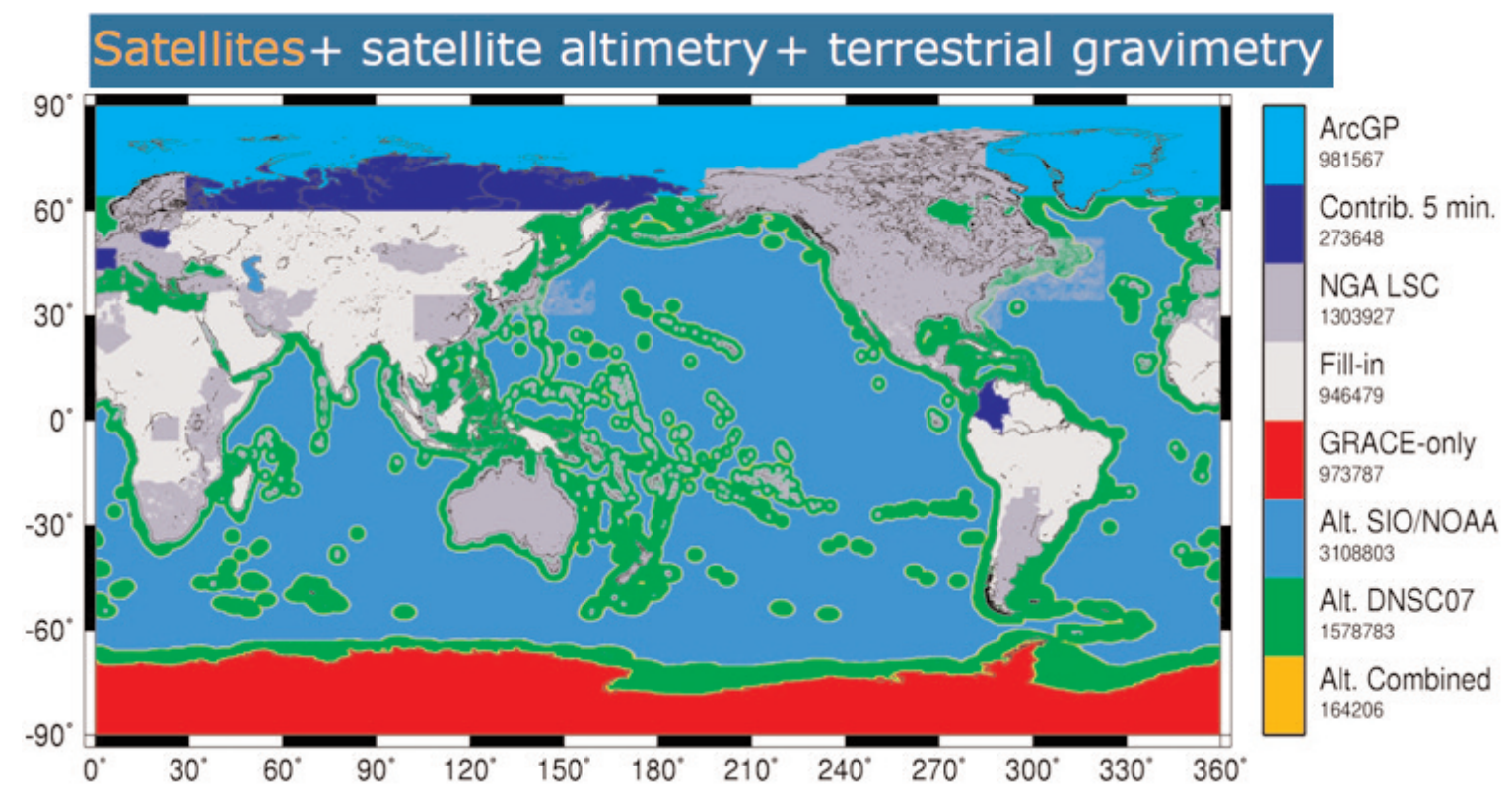

Hình 2: Các nguồn dữ liệu trọng lực dùng để tính toán các hệ số điều hòa cầu [1]

Như chúng ta thấy trên hình 2 , ở khu vực biển và ven biển từ vĩ độ -70 đến 60 sử dụng dữ liệu đo cao vệ tinh. Khu vực bắc Mỹ, Australia, Tây âu, Mông cổ, Nhật bản và 1 phần Trung quốc và Nam mỹ, Nam phi là các khu vực có dữ liệu được cung cấp chính thức từ các dự án trọng lực mặt đất. Trong khi đó khu vực đất liền của Việt Nam dữ liệu trọng lực đưa vào tính toán là các dữ liệu được thu thập từ các nguồn không chính thức (Phần lớn là dữ liệu trọng lực vệ tinh đo ở phần đất liền nên có độ chính xác không cao và không tin 
cậy, ký hiệu là Fill-in) có tác dụng lấp đầy các khoảng trống để đảm bảo miền dữ liệu đầu vào là liên tục trên các ô trọng lực chuẩn 5'x5'.

Như vậy mặc dù mô hình có bậc tối đa là 2190 nhưng với các khu vực có dữ liệu đầu vào dạng Fill-in như phần đất liền của Việt Nam các hệ số điều hòa cầu cũng chỉ có ý nghĩa ở bước sóng dài tương đương với dữ liệu trọng lực vệ tinh để xác định các hệ số điều hòa cầu tới bậc 180, các bậc tiếp theo chỉ có ý nghĩa với các khu vực có kết hợp với dữ liệu mặt đất có độ chính xác và mức độ chi tiết cao, mặc dù hiện nay các khu vực Fill-in đã được bổ sung thêm các dữ liệu từ vệ tinh GOCE nhưng độ chính xác cũng chỉ được cải thiện cho phần biển.

Trong bài báo khoa học này, chúng tôi sẽ nghiên cứu và thực nghiệm để làm rõ vấn đề: Khi chưa sử dụng các dữ liệu đo trọng lực chi tiết ở Việt Nam để xây dựng mô hình các hệ số điều hòa cầu, thì mô hình này chỉ cho phép xác định dị thường độ cao đến bậc khai triển điều hòa bao nhiêu là có ý nghĩa?

\section{Giải quyết vấn đề}

Như chúng ta đã biết công thức tính thế nhiễu tại 1 điểm trên bề mặt Trái đất từ các hệ số điều hòa cầu như sau:

$$
T(r, \theta, \lambda)=\frac{G M}{r} \sum_{l=2}^{\infty}\left(\frac{a}{r}\right)^{l} \sum_{m=0}^{l}\left(\bar{C}_{l m} \cos m \lambda+\bar{S}_{l m} \sin m \lambda\right) \bar{P}_{l m}(\cos \theta)
$$

Từ công thức trên suy ra công thức tính giá trị dị thường trọng lực tại 1 điểm trên bề mặt Trái đất từ các hệ số điều hòa cầu như sau:

$$
\Delta g(r, \theta, \lambda)=\frac{G M}{r^{2}} \sum_{l=2}^{\infty}(l-1)\left(\frac{a}{r}\right)^{l} \sum_{m=0}^{l}\left(\bar{C}_{l m} \cos m \lambda+\bar{S}_{l m} \sin m \lambda\right) \bar{P}_{l m}(\cos \theta)
$$

Công thức tính giá trị dị thường độ cao tại 1 điểm trên bề mặt Trái đất từ các hệ số điều hòa cầu như sau:

$$
N(r, \theta, \lambda)=\frac{G M}{r \gamma} \sum_{l=2}^{\infty}\left(\frac{a}{r}\right)^{l} \sum_{m=0}^{l}\left(\bar{C}_{l m} \cos m \lambda+\bar{S}_{l m} \sin m \lambda\right) \bar{P}_{l m}(\cos \theta)
$$

Trong đó:

$-r, \theta, \lambda$ : là tọa độ điểm xét trên Ellipsoid quy chiếu.

- a: là bán trục lớn Ellipsoid quy chiếu.

- GM: là hằng số trọng trường địa tâm của Trái đất.

- $\gamma$ : là gia tốc trọng trường chuẩn tại vị trí điểm xét.

- I, m: là bậc và mức của hệ số điều hòa cầu.

- $\bar{C}_{l m}, \bar{S}_{l m}$ : là các hệ số điều hòa cầu được chuẩn hóa.

- $\bar{P}_{l m}(\cos \theta)$ : Hàm Legendre được chuẩn hóa (normalized Legendre associated functions) 
Để tính $\bar{P}_{l m}(\cos \theta)$ chúng ta sử dụng công thức truy hồi như sau:

$$
\bar{P}_{l m}(\cos \theta)=W_{l m}\left[(\cos \theta) \bar{P}_{l-1, m}(\cos \theta)-W_{l-1, m}^{-1} \bar{P}_{l-2, m}(\cos \theta)\right]
$$

Trong đó: $\bar{P}_{0,0}=0$

\begin{tabular}{|l|l|}
\hline Với l=1 & $W_{1,1}=\sqrt{3}$ \\
\hline Với $\mathbf{l}>\mathbf{1}$ & $W_{l, l}=\sqrt{\frac{2 l+1}{2 l}}$ \\
\hline Và & $W_{l, m}=\sqrt{\frac{(2 l+1)(2 l-1)}{(l+m)(l-m)}}$ \\
\hline
\end{tabular}

Qua các công thức trên chúng ta thấy rằng việc lập trình xử lý tính toán là hết sức phức tạp, trong phần thực nghiệm này các phương pháp tính toán hiện đại đã được chúng tôi nghiên cứu đưa vào sử dụng đó là phương pháp truy hồi và một số phương pháp biến đổi ma trận khác để chương trình chạy nhanh, giảm thiểu các sai số tính toán khi tập dữ liệu hệ số điều hòa cầu bậc cao là rất lớn.

Mối quan hệ giữa bậc tối đa và bước sóng trung bình của mô hình như sau:

- Công thức gần đúng: $l_{\max }=\frac{\pi R}{\psi_{t b}}$ suy ra $\psi_{t b}=\frac{\pi R}{l_{\max }}$

- Công thức chính xác $\psi_{t b}=4 \arcsin \left(\frac{1}{l_{\max }+1}\right)$

Ví dụ: với I $\max =180$ suy ra $\psi_{t b}=1.266^{\circ}$

Trong trường hợp nếu bổ sung dữ liệu trọng lực mặt đất thì mật độ (khoảng cách) trung bình của dữ liệu sẽ quyết định việc cho phép tính được các hệ số điều hòa cầu đến bậc tối đa thứ bao nhiêu là có ý nghĩa.

Trên cơ sở nghiên cứu lý thuyết và các công thức tính toán như trên, chúng tôi tiến hành lập trình thực nghiệm khảo sát tính toán giá trị dị thường độ cao tại một số khu vực đặc trưng ở Việt Nam (trong đất liền và ở vùng Biển Đông Việt Nam), các giá trị dị thường độ cao được tính toán theo sự thay đổi bậc tối đa của các hệ số điều hòa cầu từ 2 đến 360 . Kết quả như sau: (Xem hình 3)

Qua biểu đồ hình 3 cho phép chúng ta kiểm chứng rằng khi các hệ số điều hòa được tính toán mà không sử dụng dữ liệu đo trọng lực chi tiết ở Việt Nam và chỉ sử dụng các dữ liệu trọng lực vệ tinh với dữ liệu trọng lực trung bình trên các ô chuẩn có kích thước lớn, dẫn đến khi dùng các hệ số điều hòa này để tính toán ngược trở lại các giá trị dị thường trọng lực và dị thường độ cao thì chỉ đến bậc 180 là có ý nghĩa (Các bậc cao hơn làm thay đổi giá trị nhỏ không tin cậy, do ảnh hưởng của dữ liệu trọng lực mặt đất từ các khu vực khác). Bậc tối đa 180 có nghĩa là mô hình có bước sóng dài là $1.266^{\circ}$ theo dữ liệu vệ tinh. Điều này hoàn toàn phù hợp với các phân tích lý thuyết ở trên. 


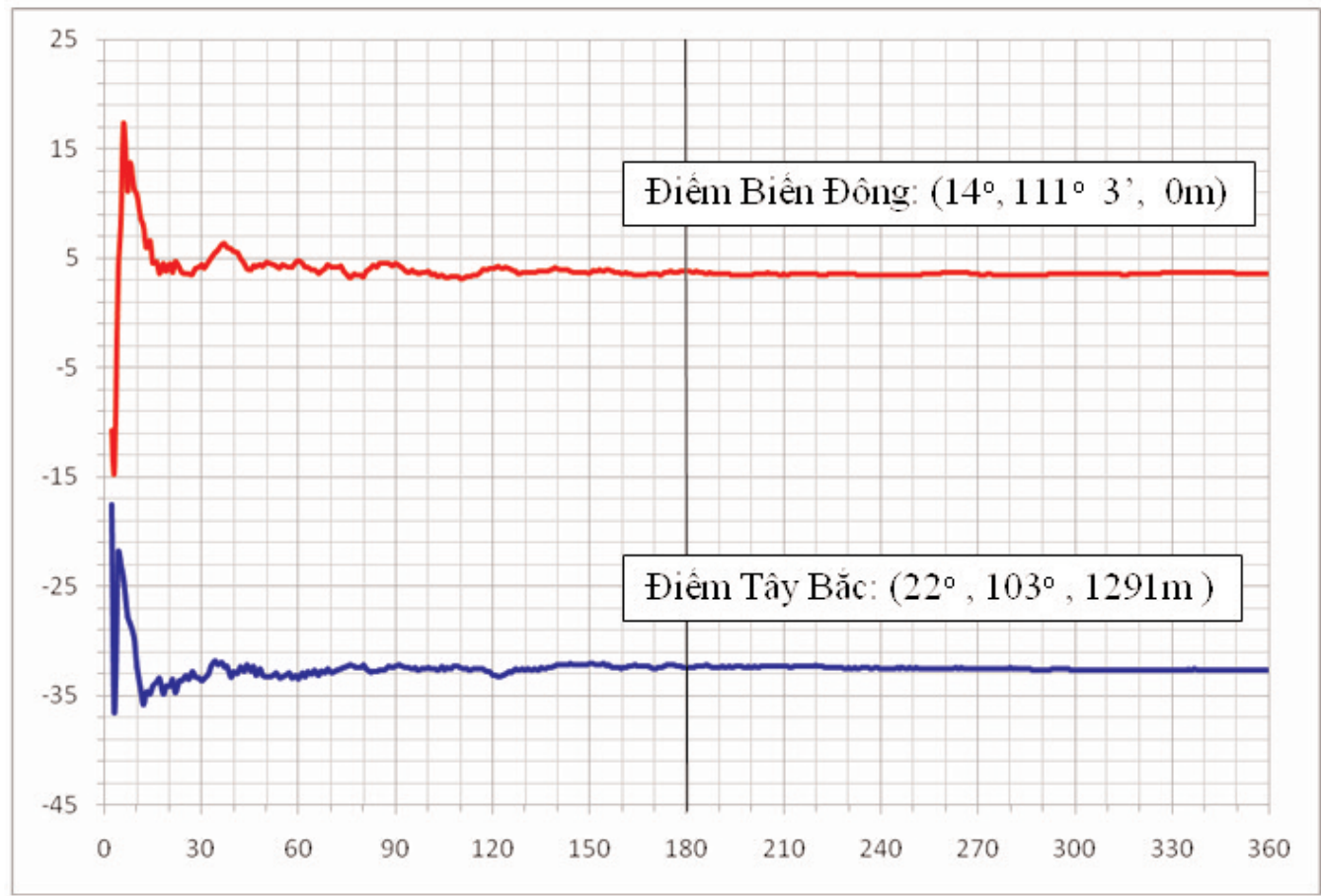

Hình 3: Biểu đồ sụ̣ thay đổi giá trị dị thường độ cao theo sự thay đổi bậc của hệ số điều hòa cầu tại 2 điểm đặc trưng

\section{Kết luận}

Bài báo đã phân tích các nguồn dữ liệu dùng để xây dựng mô hình hệ số điều hòa cầu, nghiên cứu sử dụng các công thức phù hợp, qua đó xây dựng thuật toán để lập trình tính toán thực nghiệm giá trị dị thường trọng lực và dị thường độ cao tại khu vực Việt Nam từ các hệ số điều hòa cầu.

Kết quả tính toán thực nghiệm trên các điểm ở Việt Nam đã cho phép khẳng định chắc chắn các cơ sở lý thuyết đã nghiên cứu và chỉ ra rằng ở khu vực Việt Nam cũng như một số khu vực khác dữ liệu trọng lực tham gia ở mức độ Fill-in vào quá trình tạo ra các hệ số điều hòa cầu thì mặc dù bậc của hệ số điều hòa cầu công bố có cao đến đâu thì cũng chỉ đến bậc 180 là có ý nghĩa để tính toán cho khu vực Fill-in mà thôi. Điều này hoàn toàn phù hợp với các hệ số khai triển điều hòa bậc thấp (tương đương với mô hình bước sóng dài) được xác định từ dữ liệu trọng lực vệ tinh.

Để hoàn thiện các hệ số điều hòa cầu bậc cao tính toán được mô hình có mật độ cao, bước sóng ngắn ở khu vực Việt Nam chúng ta cần nghiên cứu xây dựng giải pháp bổ sung dữ liệu trọng lực mặt đất để tính toán cải chính lại các hệ số điều hòa cầu đã có. $O$

\section{Tài liệu tham khảo}

[1]. Nikolaos K. Pavlis, Simon A. Holmes, Steve C. Kenyon, and John K. Factor (2008), An Earth Gravitational Model to Degree 2160: EGM2008, EGU General Assembly, 2008, Vienna, Austria. 